\title{
ERRATUM
}

\section{Energy dissipation caused by boundary layer instability at vanishing viscosity - ERRATUM}

\author{
Natacha Nguyen van yen, Matthias Waidmann, Rupert Klein, Marie Farge \\ and Kai Schneider
}

doi:10.1017/jfm.2018.396, Published by Cambridge University Press, 26 June 2018

In the original version of this article (Nguyen van yen et al. 2018), the copyright statement incorrectly indicated that it had been transferred to Cambridge University Press. The authors retain the copyright of this work. The copyright line has been updated in the original to the following:

(c) The Authors 2018

The publisher apologises to the authors for this mistake.

\section{REFERENCE}

Nguyen van yen, N., Waidmann, M., Klein, R., Farge, M. \& Schneider, K. 2018 Energy dissipation caused by boundary layer instability at vanishing viscosity. J. Fluid Mech. 849, 676-717, doi:10.1017/jfm.2018.396. 